\title{
Bi-Layered Disulfiram-Loaded Fiber Membranes With Antibacterial Properties for Wound Dressing
}

\section{Chenchen Xie}

Changchun University of Science and Technology

Jin Yan

Changchun University of Science and Technology

\section{Siyuan Cao}

Changchun University of Science and Technology

Ri Liu

Changchun University of Science and Technology

\section{Baishun Sun}

Changchun University of Science and Technology

\section{Ying Xie}

Changchun University of Science and Technology

\section{Kaige Qu}

Changchun University of Science and Technology

\section{Wenxiao Zhang}

Changchun University of Science and Technology

\section{Zhankun Weng}

Changchun University of Science and Technology

\section{Zuobin Wang ( $\nabla$ wangz@cust.edu.cn )}

Changchun University of Science and Technology

\section{Research Article}

Keywords: polylactic acid (PLA), PVDF, Gram-negative, wound dressings application

Posted Date: June 3rd, 2021

DOI: https://doi.org/10.21203/rs.3.rs-535473/v1

License: (c) (1) This work is licensed under a Creative Commons Attribution 4.0 International License. Read Full License 
Version of Record: A version of this preprint was published at Applied Biochemistry and Biotechnology on October 29th, 2021. See the published version at https://doi.org/10.1007/s12010-021-03663-0. 


\section{Bi-layered disulfiram-loaded fiber membranes with antibacterial properties}

2 for wound dressing

3 Chenchen Xie ${ }^{a, b}$, Jin Yan ${ }^{a, b}$, Siyuan Cao ${ }^{a, b}$, Ri Liu ${ }^{\text {a,b }}$, Baishun Sun ${ }^{a, b}$, Ying Xie ${ }^{a, b}$, Kaige Qu

4 a,b, Wenxiao Zhang ${ }^{\text {a,b }}$, Zhankun Weng ${ }^{\text {a,b }}$, Zuobin Wang a,b,c,*

5 a International Research Centre for Nano Handing and Manufacturing of China (CNM),

6 Changchun University of Science and Technology, Changchun 130022, China.

7 b Ministry of Education Key Laboratory for Cross-Scale Micro and Nano Manufacturing,

8 Changchun University of Science and Technology, Changchun 130022, China.

${ }^{\mathrm{c}}$ IRAC \& JR3CN, University of Bedfordshire, Luton LU1 3JU, UK.

10 Keywords: Bi-layered disulfiram-loaded fiber membranes; Antibacterial activity; Surface 11 wettability; Electrospinning; Wound dressing

\section{Abstract}

In this study, the bi-layered disulfiram-loaded fiber membranes with antibacterial activity and different surface wettabilities are prepared using electrospinning technology. In the application of wound dressing, the hydrophilic surface of fiber membranes is beneficial for the cell adhesion and drug release to heal the wound, meanwhile the hydrophobic outside surface is able to block water penetration to reduce the probability of wound infection. The obtained bi-layered drug-loaded fiber membranes are composed of polyvinylidene fluoride (PVDF) bottom surface and disulfiram (DSF)/polylactic acid (PLA) top surface. To modify the top surface wettability, the oxygen plasma modification of bi-layered membranes was carried out. We analyzed the morphology, wettability and chemical compositions of bilayered drug-loaded fiber membranes by various techniques. And the bi-layered disulfiramloaded membranes showed potent antibacterial activity in vitro against both Escherichia coli

\footnotetext{
* To whom correspondence should be addressed. E-mails: wangz@cust.edu.cn.
} 
1 (Gram-negative) and Staphylococcus aureus (Gram-positive). Thus, the obtained bi-layered

2 disulfiram-loaded fiber membranes are very suitable for wound dressings application. 


\section{1. Introduction}

Fiber membranes are widely applied in biomedicine used as wound dressings, tissue scaffold and medicine carrier. [1-3] The fiber membranes consist of various fibers with different diameters. In the application of wound dressing, it would avoid physical wound damages and balance wound microenvironments, meanwhile the membranes can serve as the drug carrier to promote the wound healing. [4,5] Therefore, drug-loaded fiber membranes prepared by biocompatible materials have become more and more popular as wound dressings. Among several approaches to prepare composite fiber membranes, electrospinning is considered to be the most practicable and helpful technique. [6] The electrospinning fiber membranes with 3D reticulate structure are very similar to the configuration of natural extracellular matrix (ECM), so it would provide suitable support site for cell adhesion and proliferation. [7] As wound dressings, the high specific surface area of electrospinning fiber membranes is conducive to absorb the wound exudate and release drugs. The small pore formed by interlacing of fibers ensures the gas exchange, meanwhile it would avoid the invasion of external liquids and bacteria effectively to protect the wound from infection. Moreover, electrospinning process is simple, flexible and cost-effective. [8] According to the actual situation of the wound, electrospinning fiber membranes can be incorporated various drugs flexibly, such as antibacterial agents, vitamins and growth factors, to further promote wound healing. [9, 10] Therefore, electrospinning composite membranes have been widely used in wound healing. [11, 12]

In addition, it is noteworthy that the surface wettabilities of wound dressing are vital. [13] The hydrophilic surface of the wound dressing adheres to the wound directly, which is 
1 conducive to the cell adhesion and drug release to treat the wound, meanwhile the

2 hydrophobic surface would block the penetration of water containing microorganisms to

3 protect the wound from infection. [7] Thus, the membranes with different wettability surfaces

4 have great potential in wound dressing applications. Nevertheless, the traditional

5 electrospinning membranes are single layer with the same wettability on both surfaces.

6 Therefore, it is desirable to develop bi-layered fiber membranes with different wettability

7 surface layers to optimize the wound treatment.

Fiber membranes with antibacterial activity have gained much interest as wound

9 dressings. [14] Disulfiram (DSF) is an old oral drug for abstinence from alcohol. [15] Lately,

10 there are several approaches reporting that the DSF possesses the potent antimicrobial activity.

11 [16-18] Notably, the repurposing of DSF is hopeful for reducing the drug-resistance of

12 bacteria. However, DSF would degrade rapidly in the acidic gastric juice and bloodstream.

13 Thus, the poor water solubility and physiological instability of DSF obviously restricts its

14 practical clinical application. [19-22]

In this study, we developed a disulfiram-loaded scaffold using the electrospinning method to enhance the stability of DSF and to facilitate its appropriate distribution. To be specific, the bi-layered drug-loaded fiber membranes were composed of a PVDF bottom layer and a DSF/PLA top layer. The morphology, wettability and chemical components of the obtained membranes were measured by scanning electronic microscope (SEM), drop shape analysis, X-ray diffractometer (XRD) and X-ray photoelectron spectrometer (XPS). The bacteriostatic ability of the bi-layered drug-loaded fiber membranes was verified against 
1 results indicate that the bi-layered drug-loaded fiber membranes are very suitable for

2 application in the field of wound dressings.

\section{Experimental methods}

\subsection{Materials}

Polyvinylidene fluoride (PVDF, $M_{\mathrm{w}} \approx 543000 \mathrm{~g} / \mathrm{mol}$ ) powder and disulfiram (DSF) powder were purchased from Sigma-Aldrich. Polylactic acid (PLA, 4032D, $M_{\mathrm{w}} \approx 160000$ $\mathrm{g} / \mathrm{mol}$ ) powder was purchased from NatureWorks LLC. All other analytically pure chemicals were acquired from Beijing Chemical Works. Escherichia Coli (E. Coli) and Staphylococcus aureus (S. Aureus) were supplied by the local laboratory.

\subsection{Preparation of bi-layered membranes}

The bi-layered drug-loaded fiber membranes composed of the bottom layer PVDF fibers and top layer DSF/PLA fibers were fabricated using ths electrospinning method. In brief, the PVDF powder was added in dimethylformamide (DMF) /acetone $(1: 1, \mathrm{v} / \mathrm{v})$ solvent, then the obtained PVDF solution with $21 \mathrm{wt} \%$ was stirred at $40{ }^{\circ} \mathrm{C}$ for $12 \mathrm{~h}$. The DSF/PLA $(1: 5, \mathrm{w} / \mathrm{w})$ blended solution was obtained by adding the DSF powder in the DMF/acetone $(1: 1, \mathrm{v} / \mathrm{v})$ solution of PLA (12.5 wt\%) and stirring for $12 \mathrm{~h}$ at $40{ }^{\circ} \mathrm{C}$. During electrospinning, the flow rate of spinning solution was kept on $0.5 \mathrm{ml} / \mathrm{h}$. A 27 -gauge steel needle was connected to an positive voltage of $13 \mathrm{kV}$. A grounded $\mathrm{Al}$ foil was applied as the collector. And the distance between the needle and collector was about $14 \mathrm{~cm}$. PVDF fibers were collected on Al foilcovered plate as the bottom layer and the subsequent DSF/PLA fibers were directly deposited on the PVDF layer. The collected bi-layered fiber membranes were completely dried at room 
1 temperature overnight before further use. The obtained bi-layered fiber membranes are

2 denoted as PLA-PVDF and DSF/PLA-PVDF.

\subsection{Plasma treatment of bi-layered membranes}

The oxygen plasma modification of bi-layered drug-loaded fiber membranes was carried out in a $13.56 \mathrm{MHz}$ RF power generator (Diener Electronic Pico, Germany). The maximum power of the generator is $300 \mathrm{~W}$. The oxygen partial pressure was precisely adjusted to 0.3 mbar. All plasma treatments were performed for $40 \mathrm{~s}$ at $30 \%$ of the maximum power. The modified sample was denoted as plasma-treated DSF/PLA-PVDF.

\subsection{Characterization of bi-layered membranes}

The morphological features of bi-layered membranes were examined using SEM (FEI QUANT-250 FEG, USA) at an operating voltage of $7 \mathrm{kV}$. The elementary compositions of bilayered membranes were analyzed by an energy dispersive spectroscope (EDS, Oxford Instruments, UK). The degree of crystallinity of samples was measured by X-ray diffraction (XRD) with $\mathrm{Cu} / \mathrm{K}_{\alpha}$ radiation at $30 \mathrm{kV}$ and $200 \mathrm{~mA}$.

Water contact angles (WCAs) of the bottom and top surface layers of bi-layered membranes were measured by drop shape analysis (Kruss DAS100, Germany) at room temperature. The WCA value of water drop $(5 \mu 1)$ was tested after the droplet was stable. The chemical compositions of the bi-layered membranes before and after plasma modifications were studied by X-ray photoelectron spectrometer (XPS, Thermo Fisher Scientific, USA).

\subsection{Antibacterial activity}

The antibiotic ability of the bi-layered membranes was tested based on agar plate spreading susceptibility. The inhibition zones were detected against a representative Gram- 
1 negative bacteria E. Coli and a representative Gram-positive bacteria S. Aureus. The bi-

2 layered membranes were divided into round plate (diameter $=15 \mathrm{~mm})$ and exposed under UV

3 light for $1 \mathrm{~h}$ to sterilize. About $100 \mu \mathrm{L}\left(10^{5} \mathrm{CFU}\right)$ of each bacteria was cultured on the agar

4 medium. Within each culture-medium, three types of membranes (PLA-PVDF, DSF/PLA-

$5 \quad$ PVDF and plasma-treated DSF/PLA-PVDF) were placed on the surfaces, and then incubated

6 at $37{ }^{\circ} \mathrm{C}$ overnight. The bacteriostatic circles around the membranes were measured to

7 evaluate the antibacterial activity against the test organism.

\section{Results and discussions}

The bi-layered drug-loaded fiber membranes were prepared using two step electrospinning process as stated in Fig. 1. In brief, PVDF membranes were firstly fabricated on an aluminum foil. Then the obtained PVDF membranes with the aluminum foil acted as a collector to collect the drug-loaded PLA fiber membranes. At last, the fabricated bi-layered membranes were modified by oxygen plasma treatment to form the plasma-treated DSF/PLAPVDF bi-layered membranes.

\subsection{Surface morphology of bi-layered membranes}

The top (PLA) and bottom (PVDF) surface morphologies of bi-layered membranes were obtained by SEM. As shown in Fig. 2, there were not remarkable differences among the PLAPVDF, DSF/PLA-PVDF and plasma-treated DSF/PLA-PVDF in the surface morphologies. The bottom surface PVDF fibers mainly ranged from 500 to $980 \mathrm{~nm}$. Furthermore, it can be scanned that the morphologies of PLA fibers or drug-loaded DSF/PLA fibers were relatively smooth without beads, which showed that the DSF was compatible with the PLA polymer. The size distribution of top surface fibers was from 150 to $400 \mathrm{~nm}$, in the range of collagen 
1 fibers (50 to $500 \mathrm{~nm}$ ) in thenative extracellular matrix. [23] So, the obtained bi-layered

2 membranes were similar to ECM in some structural features. It was beneficial for cell

3 adhesion, proliferation and differentiation to improve the vulnus healing. [24]

\subsection{EDS and XRD Analysis}

The EDS spectra of the PLA-PVDF, DSF/PLA-PVDF and plasma-treated DSF/PLAPVDF are shown in Fig. 3(a). As observed in Fig. 3(a), the characteristic peak of sulfur (S) in the EDS spectra of drug-loaded membranes verifies that the drug exists on the surface of the DSF/PLA-PVDF and plasma-treated DSF/PLA-PVDF. It demonstrates that the bi-layered drug-loaded membranes succeed in incorporating DSF using the electrospinning method.

Figs. 3(b) and (c) present the XRD patterns of DSF powders, PVDF fibers, PLA fibers, PLA-PVDF, DSF/PLA-PVDF and plasma-treated DSF/PLA-PVDF. From the PLA fiber pattern in Fig. 3(b), there is a broad diffusion scattering around $20^{\circ}$ which indicates that the PLA fibers are in amorphous phase. Amorphous electrospun membranes have higher cell adhesion and proliferation when compared to crystalline material. [25] Hence, the PLA fiber scaffolds as the top surface layer are highly desirable for tissue regeneration. From the patterns of PVDF fibers shown in Fig. 3(b), the reflection sum of (110) $\alpha$ and (200) $\beta$ at about 20.26 degrees is detected. The results demonstrate that the PVDF electrospinning fibers are mainly in $\beta$ phase. [26] Notably, as shown in Fig. 3(c), when PLA fibers are covered on PVDF fibers to form bi-layered membranes, the fabricated bi-layered membranes show a characteristic peak of the PVDF electrospinning membranes. Moreover, as exhibited in Fig. 3(c), the XRD patterns of the bi-layered drug-loaded membranes show a very weak 
1 representative peak at $17.38^{\circ}$ of the DSF drugs. It proves the incorporation of DSF into the bi-

2 layered membranes.

\subsection{Surface properties of bi-layered membranes}

The wettabilities of bi-layered membrane surfaces were studied by the measurement of the WCAs of top (PLA) and bottom (PVDF) surfaces. As exhibited in Fig. 4, the WCAs of PVDF and PLA surfaces are around $133.2^{\circ}$ and $126.5^{\circ}$, respectively. The water contact angles of bi-layered drug-loaded fiber membranes are around $132.1^{\circ}$ and $127.7^{\circ}$, respectively. After plasma modification, the WCAs of PLA surface layer and PVDF layer are at $54.2^{\circ}$ and $120.9^{\circ}$, respectively. The results show that PVDF and PLA are both hydrophobic materials, [7] so the untreated bi-layered membrane surfaces are hydrophobic. However, after plasma treatment, the surface of PLA fiber layer on bi-layered drug-loaded membranes is hydrophilic. It is indicated that the two surfaces of bi-layered drug-loaded membranes have different wettabilities. When the bi-layered drug-loaded membranes are applied in vulnus healing, the top hydrophilic surface adheres the wound directly and release antibacterial drugs to sterilize and heal the wound, meanwhile the bottom hydrophobic surface would block the penetration of water containing microorganisms and preserve the wound from infection.[7] Consequently, the obtained bi-layered drug-loaded fiber membranes have a broad application prospect in wound dressing.

The untreated and plasma-treated bi-layered drug-loaded fiber membranes were further analysed by XPS, as shown in Fig. 5, where C 1s peak and $\mathrm{O} 1 \mathrm{~s}$ peak are relatively apparent peaks at $284.71 \mathrm{eV}$ and $532.54 \mathrm{eV}$, respectively. In Figs. 5 (a) and (c), the $\mathrm{C} 1 \mathrm{~s}$ content of initial bi-layered drug-loaded membranes was $81.29 \%$, O 1 s was $18.71 \%$, and the $\mathrm{O} / \mathrm{C}$ atomic 
1 ratio was 0.23 . After oxygen plasma treatment, the $\mathrm{C} 1 \mathrm{~s}$ content of bi-layered membranes was

$277.98 \%$, O $1 \mathrm{~s}$ was $22.02 \%$, and the $\mathrm{O} / \mathrm{C}$ atomic ratio was increased to 0.28 . The changes in $\mathrm{C}$

3 1s peaks due to the plasma treatment are highlighted in Figs. 5 (b) and (d). The C1s line can

4 be resolved into three peaks. The three peaks are obtained at $284.8 \mathrm{eV}, 286.4 \mathrm{eV}$ and $288.9 \mathrm{eV}$,

5 matching with $\mathrm{C}-\mathrm{C}$ (nonoxygenated ring carbon), $\mathrm{C}-\mathrm{O}$ (hydroxyl and epoxy carbon), and $\mathrm{C}=\mathrm{O}$

6 (carboxyl), respectively. As shown in Fig. 5 (d), the peak strength of $\mathrm{C}-\mathrm{O}$ and $\mathrm{C}=\mathrm{O}$ increases

7 obviously after plasma treatment, which maybe due to the introduction of oxygen functional

8 groups and the increase of carboxyl and hydroxyl groups. Thus, it is also the main reason that

9 the top surface of the bi-layered membranes becomes hydrophilic.

10

11

12

\subsection{Antibacterial activity}

In order to measure the bacteriostatic ability of the bi-layered drug-loaded fiber membranes, bacteriostatic experiments were performed on Escherichia coli (Gram-negative) and Staphylococcus aureus (Gram-positive) in vitro. As exhibited in Fig. 6, there are obvious bacteriostatic circles around the drug-loaded fiber membranes. It suggests that the bi-layered drug-loaded fiber membranes show significant antibiotic ability against both E. Coli and $S$. Aureus. The bi-layered drug-loaded fiber membranes would promote the diffusion of drugs in surrounding media to protect the regenerated tissue from infection. Thus, the bi-layered drugloaded fiber membranes have the potential to be a type of good antibacterial wound dressings.

\section{Conclusions}

In summary, the bi-layered drug-loaded fiber membranes were successfully prepared by electrospinning and plasma treatment. The top and bottom surfaces of the obtained bi-layered fiber membranes had different wettabilities. The top hydrophilic surface of PLA fiber 
1 membranes would contact the wound directly to promote cell adhesion and proliferation,

2 while the outer (bottom) hydrophobic PVDF surface would prevent the penetration of water

3 containing microorganisms and preserve the wound from infection. XRD and EDS analyses

4 show that DSF is successfully incorporated into the bi-layered drug-loaded fiber membranes.

5 The antibacterial experiments suggest that the bi-layered drug-loaded fiber membranes have

6 good antibacterial effects on Staphylococcus aureus and Escherichia coli. Therefore, the

7 fabricated bi-layered drug-loaded fiber membranes have a broad application prospect in the

$8 \quad$ field of clinical wound dressing.

$9 \quad$ Ethical Approval

10 All analyses were based on previous published studies, thus no ethical approval and

11 patient consent are required.

12 Consent to Participate

$13 \quad$ Not applicable.

\section{Consent to Publish}

Not applicable.

\section{Authors Contributions}

C. Xie contributed the central idea, analysed most of the data, and wrote the initial draft of the paper. J. Yan analysed the XPS data. S. Cao performed the antibacterial activity experiment. R. Liu performed the surface wettability measurement. B. Sun analysed the XRD data. Y. Xie assisted the surface wettability measurement. K. Qu provided the materials of antibacterial activity experiment. W. Zhang provided the most of materials, reagents. Z. Weng 
1 contributed to refining the ideas and revised the manuscript. Z. Wang discussed the results

2 and revised the manuscript.

\section{$3 \quad$ Funding}

4 This work was supported by National Key R\&D Program of China 5 (No.2017YFE0112100), EU H2020 Program (MNR4SCell No.734174; NanoStencil

6 No.767285), Jilin Provincial Science and Technology Program (Nos.20180414002GH, 7 20180414081GH, 20180520203JH, 20190702002GH, and 20200901011SF), Jilin Provincial

8 DRC Research and Development Program (No.2020C022-1), and "111" Project of China 9 (No.D17017).

10 Competing Interests

11 The authors declare that they have no competing interests.

12 Availability of data and material

13 All data generated or analysed during this study are included in this published article. 


\section{References}

[1] Wang C , Wang M . Electrospun Multifunctional Tissue Engineering Scaffolds. Frontiers of Materials Science, 2014, 8(1):3-19.

[2] Zhang J G , Xiumei M O . Current research on electrospinning of silk fibroin and its blends with natural and synthetic biodegradable polymers. Frontiers of Materials Science, 2013, 7(2):129-142.

[3] Sun B , Long Y Z , Zhang H D, et al. Advances in three-dimensional nanofibrous macrostructures via electrospinning. Progress in Polymer Science, 2014, 39(5):862-890.

[4] Zhao J , Ho K K C , Shamsuddin S R, et al. A comparative study of fibre/matrix interface in glass fibre reinforced polyvinylidene fluoride composites. Colloids \& Surfaces A Physicochemical \& Engineering Aspects, 2012, 413(none):58-64.

[5] Liu S J , Chen, Liao, et al. Novel biodegradable sandwich-structured nanofibrous drugeluting membranes for repair of infected wounds: an in vitro and in vivo study. International Journal of Nanomedicine, 2012, 7:763-771.

[6] Li W, Li X, Chen Y, et al. Poly(vinyl alcohol)/sodium alginate/layered silicate based nanofibrous mats for bacterial inhibition. Carbohydr Polym, 2013, 92(2):2232-2238.

[7] Li J , Hu Y, He T, et al. Electrospun Sandwich - Structure Composite Membranes for Wound Dressing Scaffolds with High Antioxidant and Antibacterial Activity. Macromolecular Materials and Engineering, 2018:1700270.

[8] X. Li, R. Cheng, Z. Sun, W. Su, G. Pan, S. Zhao, J. Zhao, W. Cui, Flexible bipolar nanofibrous membranes for improving gradient microstructure in tendon-to-bone healing, Acta Biomater. 61 (2017) 204-216.

[9] Rieger K A , Birch N P , Schiffman J D . Designing electrospun nanofiber mats to promote wound healing - a review. Journal of Materials Chemistry B, 2013, 1(36):45314541.

[10] Unnithan A R , Barakat N A M , Pichiah P B T , et al. Wound-Dressing Materials with Antibacterial Activity from Electrospun Polyurethane-Dextran Nanofiber Mats Containing Ciprofloxacin HCl. Carbohydrate Polymers, 2012, 90(4):1786-1793. 
[11] Safdari M, Shakiba E, Kiaie S H, et al. Preparation and characterization of Ceftazidime loaded electrospun silk fibroin/gelatin mat for wound dressing. Fibers \& Polymers, 2016, 17(5):744-750.

[12] Khamforoush M , Pirouzram O , Hatami T . The evaluation of thin film composite membrane composed of an electrospun polyacrylonitrile nanofibrous mid-layer for separating oil-water mixture. Desalination, 2015, 359:14-21.

[13] Jing, Cui, Liying, et al. Co-electrospun nanofibers of PVA-SbQ and Zein for wound healing. Journal of Applied Polymer Science, 2015, 132(39).

[14] Li W , Li X , Chen Y, et al. Poly(vinyl alcohol)/sodium alginate/layered silicate based nanofibrous mats for bacterial inhibition. Carbohydr Polym, 2013, 92(2):2232-2238.

[15] Iljin, K.; Ketola, K.; Vainio, P.; Halonen, P.; Kohonen, P.; Fey, V.; Grafström, R. C.; Perälä, M.; Kallioniemi, O. High-Throughput Cell-Based Screening of 4910 Known Drugs and Drug-like Small Molecules Identifies Disulfiram as an Inhibitor of Prostate Cancer Cell Growth. Clin. Cancer Res. 2009, 150, 6070-6078.

[16] Thakare R, Shukla M, Kaul G , et al. Repurposing Disulfiram for treatment of Staphylococcus aureus infections. International Journal of Antimicrobial Agents, 2019.

[17] Horita Y , Takii T , Yagi T, et al. Antitubercular Activity of Disulfiram, an Antialcoholism Drug, against Multidrug- and Extensively Drug-Resistant Mycobacterium tuberculosis Isolates. Antimicrobial Agents \& Chemotherapy, 2012, 56(8):4140-5.

[18] Long T E . Repurposing Thiram and Disulfiram as Antibacterial Agents for MultidrugResistant Staphylococcus aureus Infections. Antimicrobial Agents \& Chemotherapy, 2017, 61(9):AAC.00898-17.

[19] Xie C, Ding R, Wang X, Hu C, Yan J, Zhang W, Wang Y, Qu Y, Zhang S, He P, Wang Z. A disulfiram-loaded electrospun poly(vinylidene fluoride) nanofibrous scaffold for cancer treatment. Nanotechnology. 2020, 31(11):115101.

[20] Xuezhi Z , Tian L , Linlin M , et al. Disulfiram-loaded mixed nanoparticles with high drug-loading and plasma stability by reducing the core crystallinity for intravenous delivery. Journal of Colloid \& Interface Science, 2018, 529:34-43. 
[21] Fasehee H , Zarrinrad G , Tavangar S M , et al. The inhibitory effect of disulfiram encapsulated PLGA NPs on tumor growth: Different administration routes. Materials Science \& Engineering C Materials for Biological Applications, 2016, 63:587-595.

[22] Songa Z T W , Bpharm T L, Wen X, et al. Stable loading and delivery of disulfiram with mPEG-PLGA/PCL mixed nanoparticles for tumor therapy. Nanomedicine Nanotechnology Biology \& Medicine, 2016, 12(2):377-386.

[23] B. Antunes, A. Moreira, V. Gaspar, I. Correia, Chitosan/arginine-chitosan polymer blends for assembly of nanofibrous membranes for wound regeneration, Carbohyd. Polym. $2015130104-112$.

[24] Chanda A , Adhikari J , Ghosh A, et al. Electrospun chitosan/polycaprolactonehyaluronic acid bilayered scaffold for potential wound healing applications. International Journal of Biological Macromolecules, 2018 116:774-785.

[25] A. Atala, R. Lanza, J.A. Thomson, R. Nerem, Principles of regenerative medicine, Academic Press 2011.

[26] Abbrent, S.; Plestil, J.; Hlavata, D. Crystallinity and morphology of PVdF-HFP-based gel electrolytes. Polymer 2001, 42, 1407-1416. 
Figures and captions
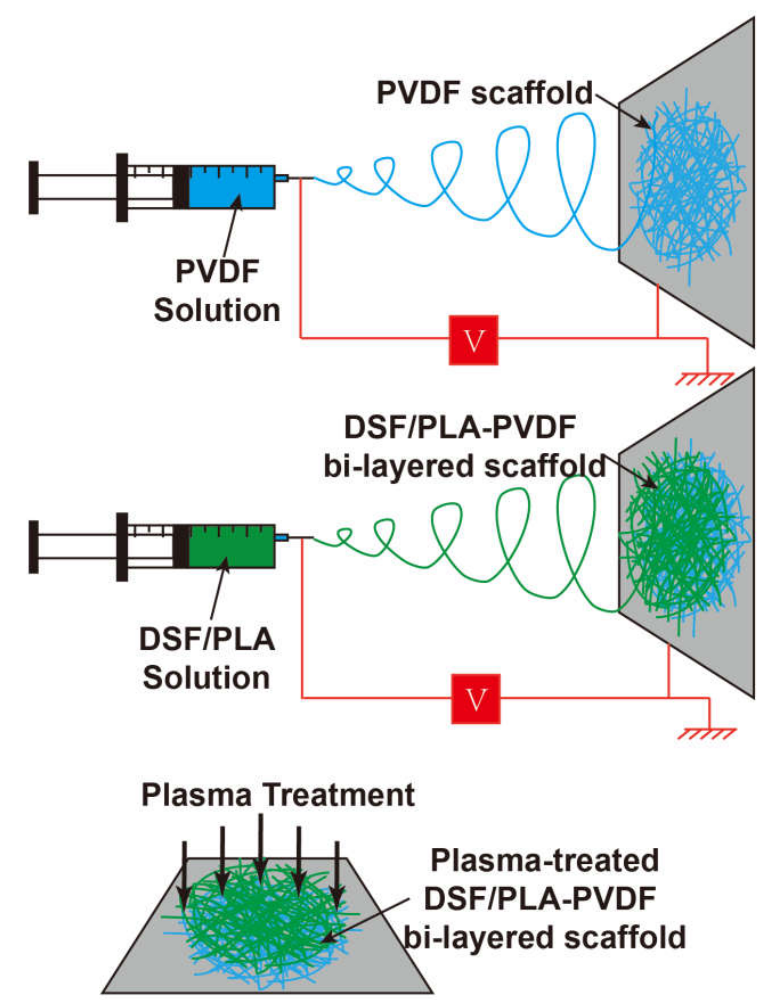

Fig. 1. Schematic diagram showing the preparation of the bi-layered drug-loaded fiber membranes. 

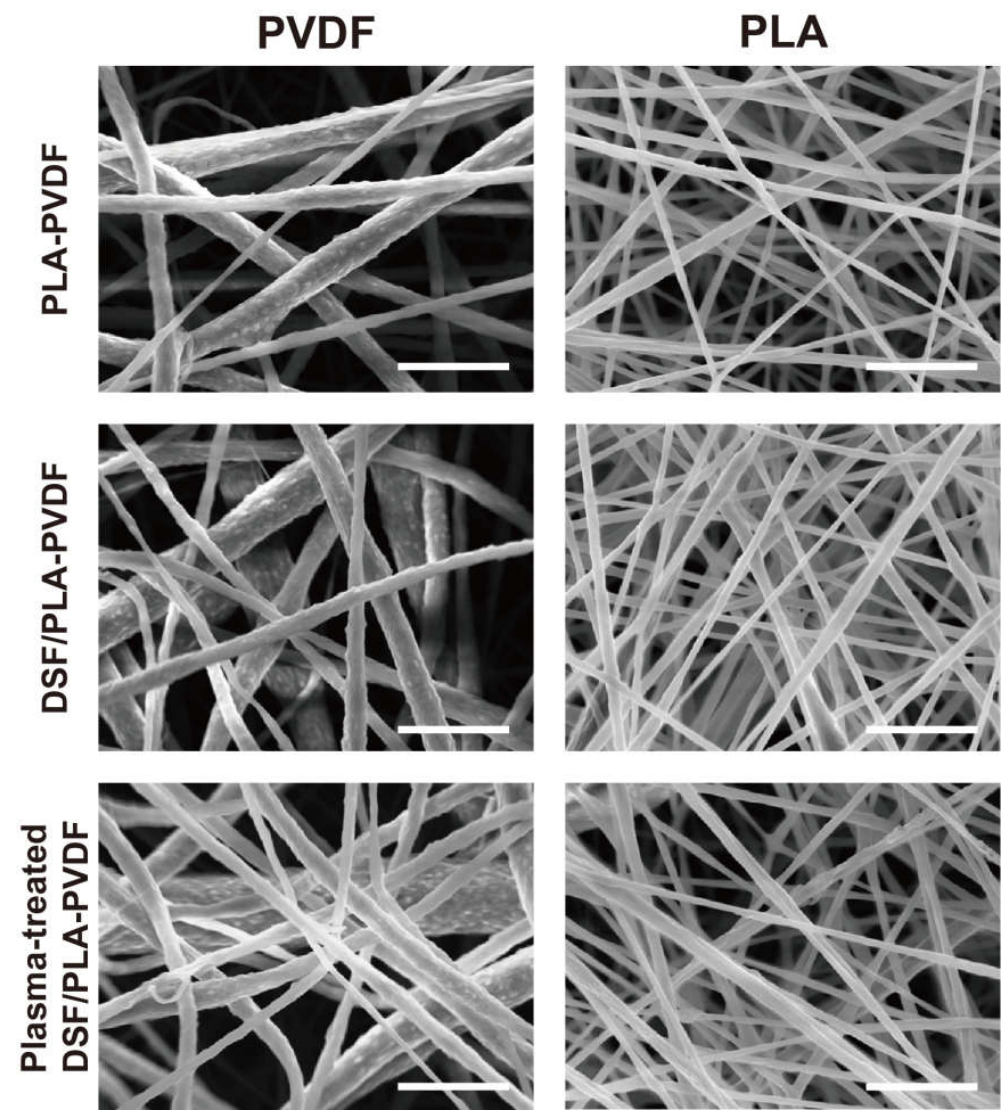

Fig. 2. The top (PLA) and bottom (PVDF) surface SEM images of PLA-PVDF, DSF/PLAPVDF and plasma-treated DSF/PLA-PVDF. (scale bar, $5 \mu \mathrm{m}$ ) 

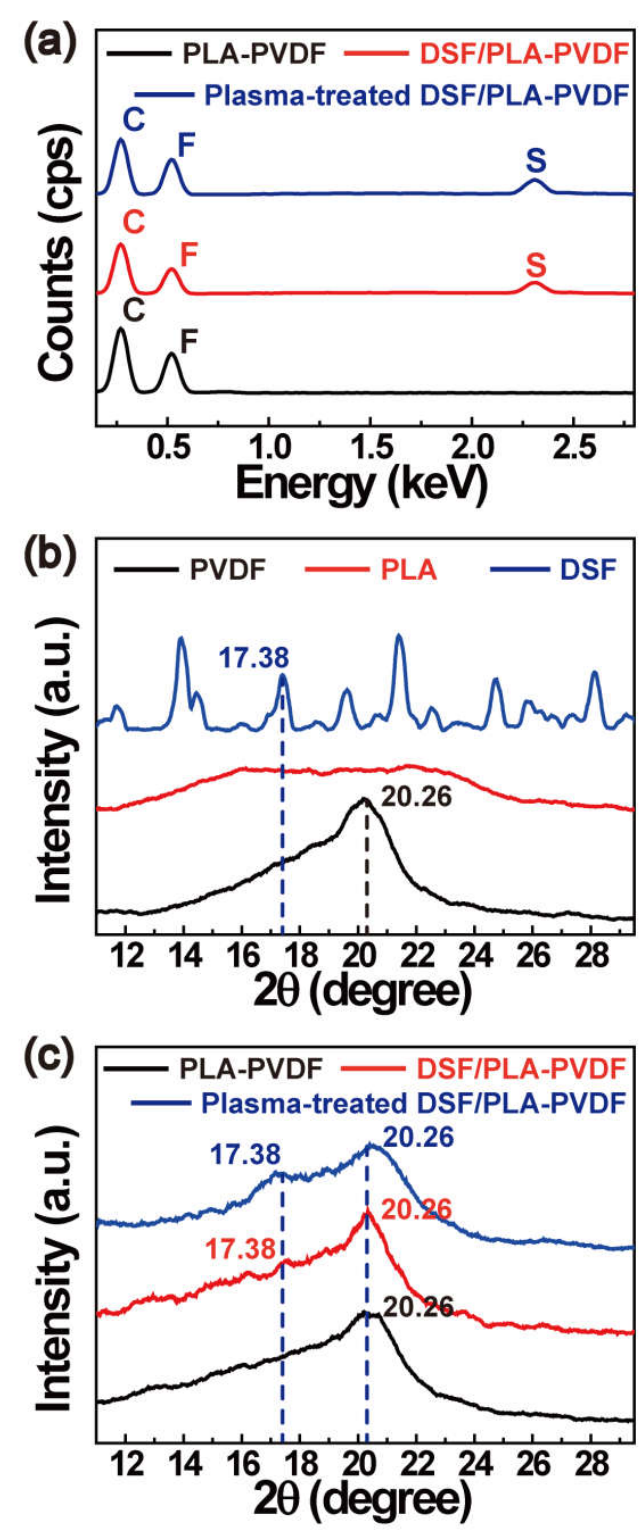

Fig. 3. (a) EDS spectrum of the bi-layered fiber membranes. (b) XRD patterns of DSF powders, PLA fibers and PVDF fibers. (c) XRD patterns of PLA-PVDF, DSF/PLAPVDF and plasma-treated DSF/PLA-PVDF. 


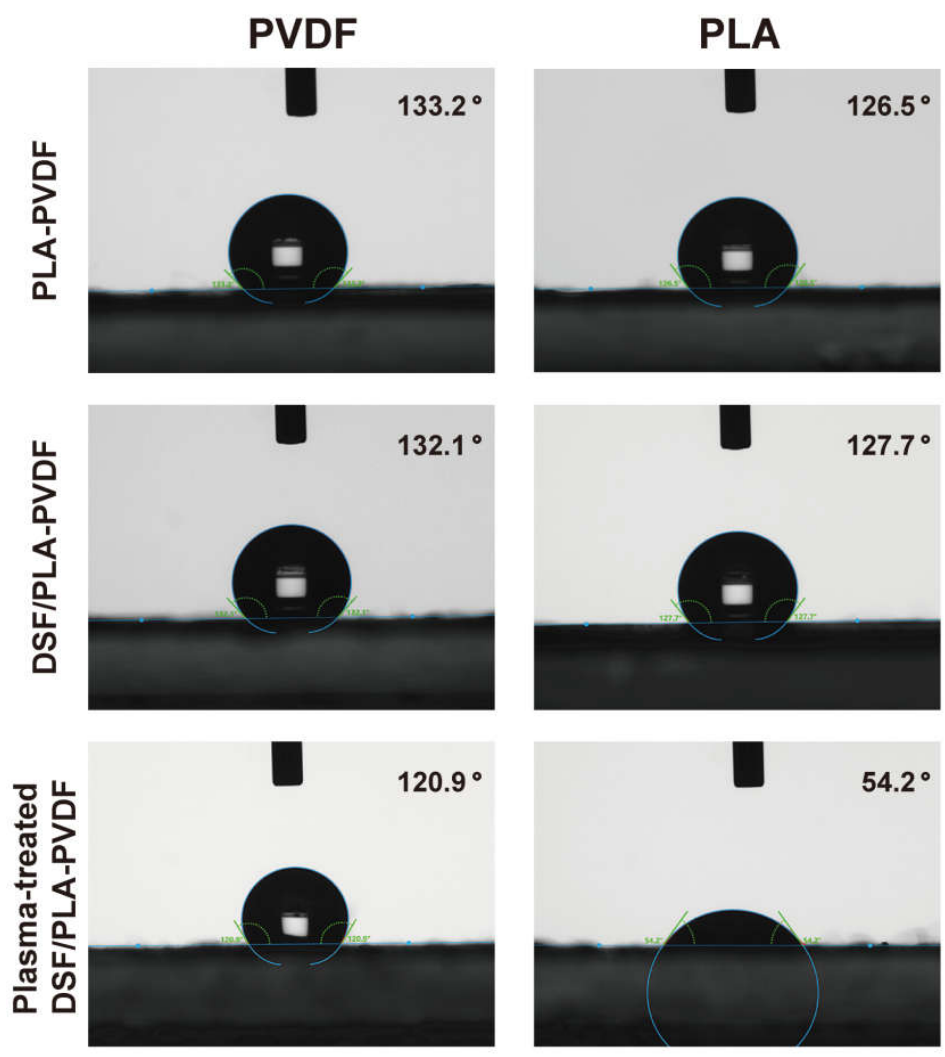

Fig. 4. The top (PLA) and bottom (PVDF) surface water contact angles of PLA-PVDF, DSF/PLA-PVDF and plasma-treated DSF/PLA-PVDF. 

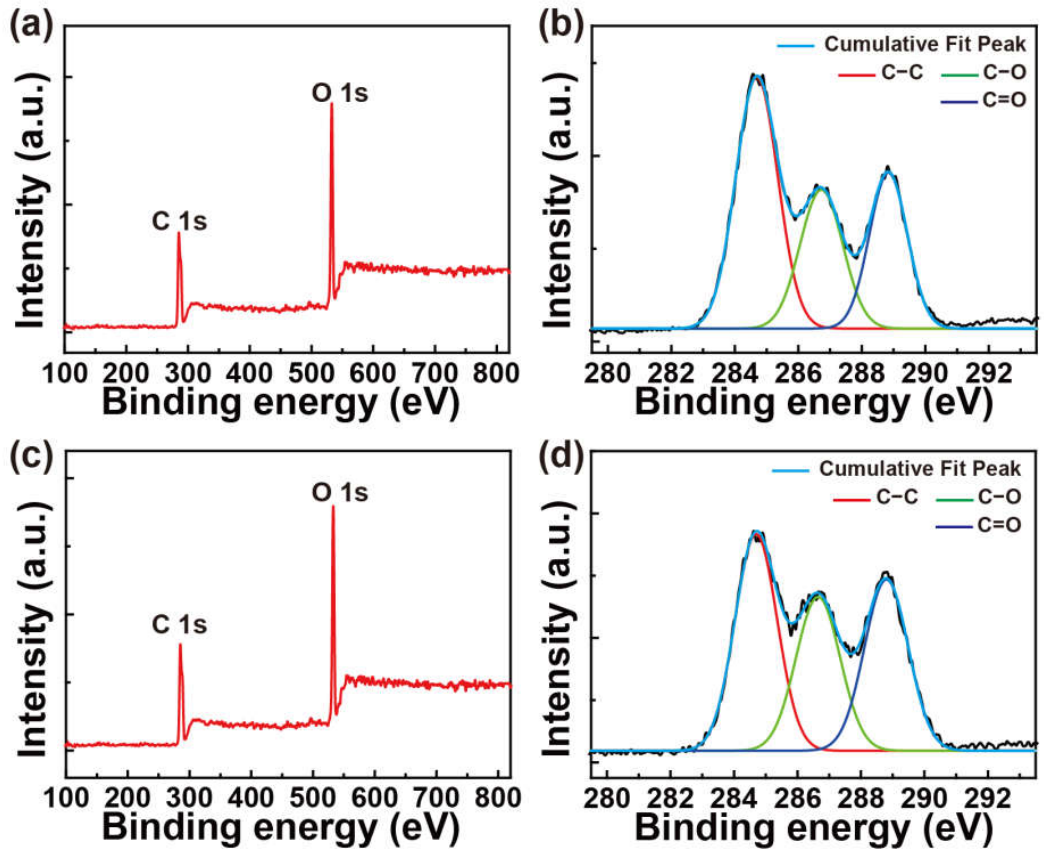

Fig. 5. (a), (b) XPS spectra survey scan and C1s XPS spectra of DSF/PLA-PVDF. (c), (d) The corresponding XPS measurement results of plasma-treated DSF/PLA-PVDF. 

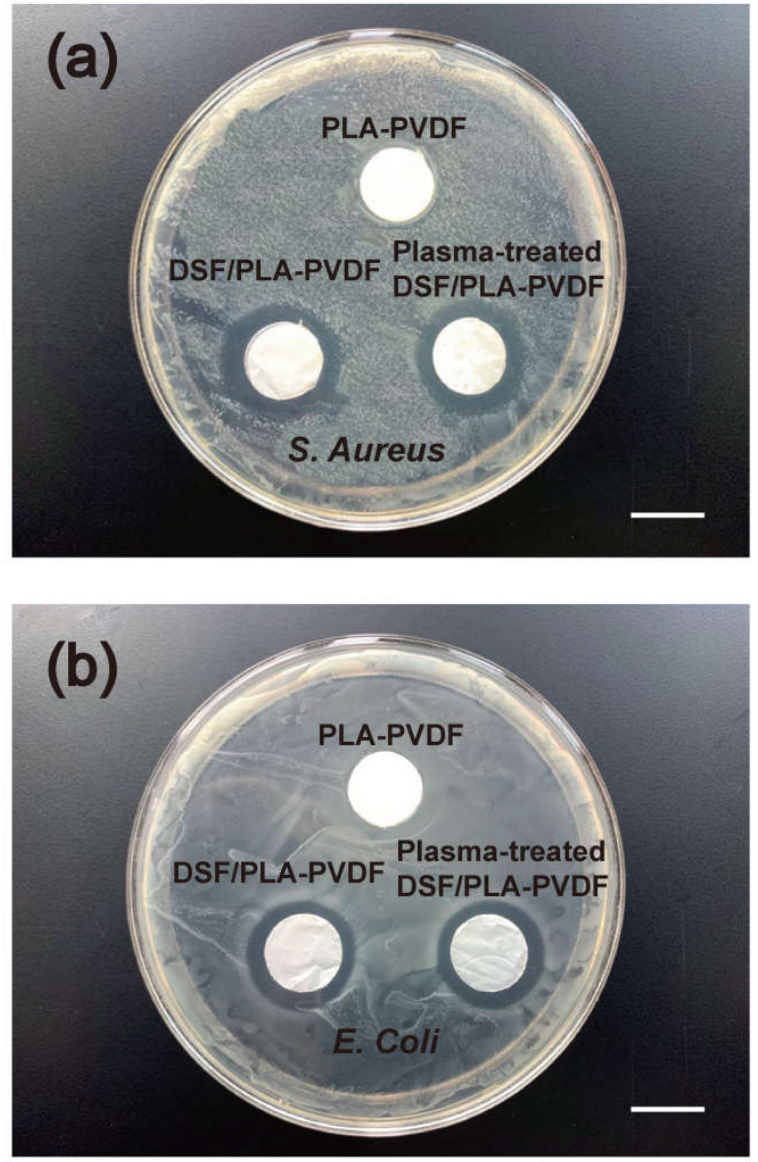

Fig. 6. Inhibition zone of bi-layered fiber membranes against (a) S. Aureus and (b) E. Coli. (scale bar, $15 \mathrm{~mm}$ ) 
Figures
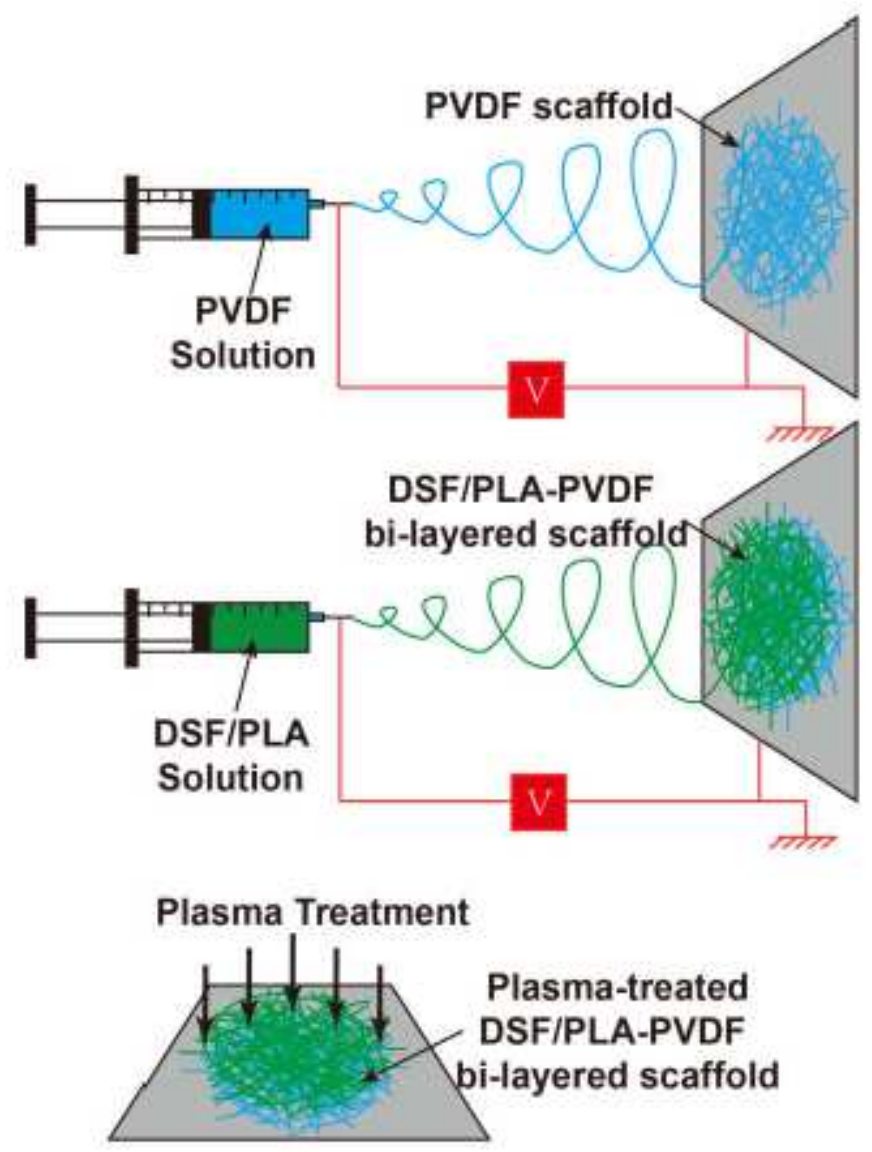

Figure 1

Schematic diagram showing the preparation of the bi-layered drug-loaded fiber membranes. 

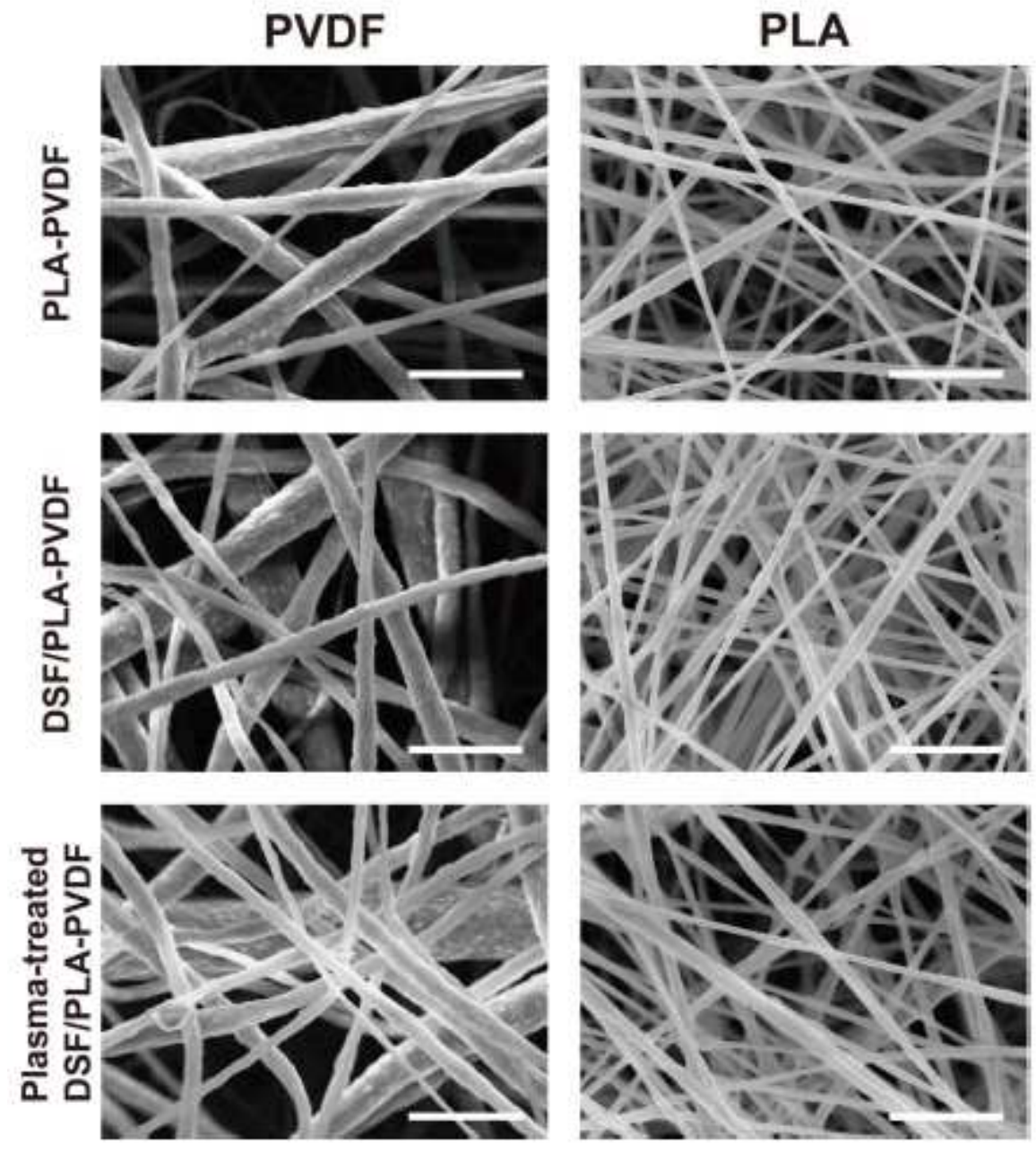

Figure 2

The top (PLA) and bottom (PVDF) surface SEM images of PLA-PVDF, DSF/PLAPVDF and plasma-treated DSF/PLA-PVDF. (scale bar, $5 \mu \mathrm{m}$ ) 

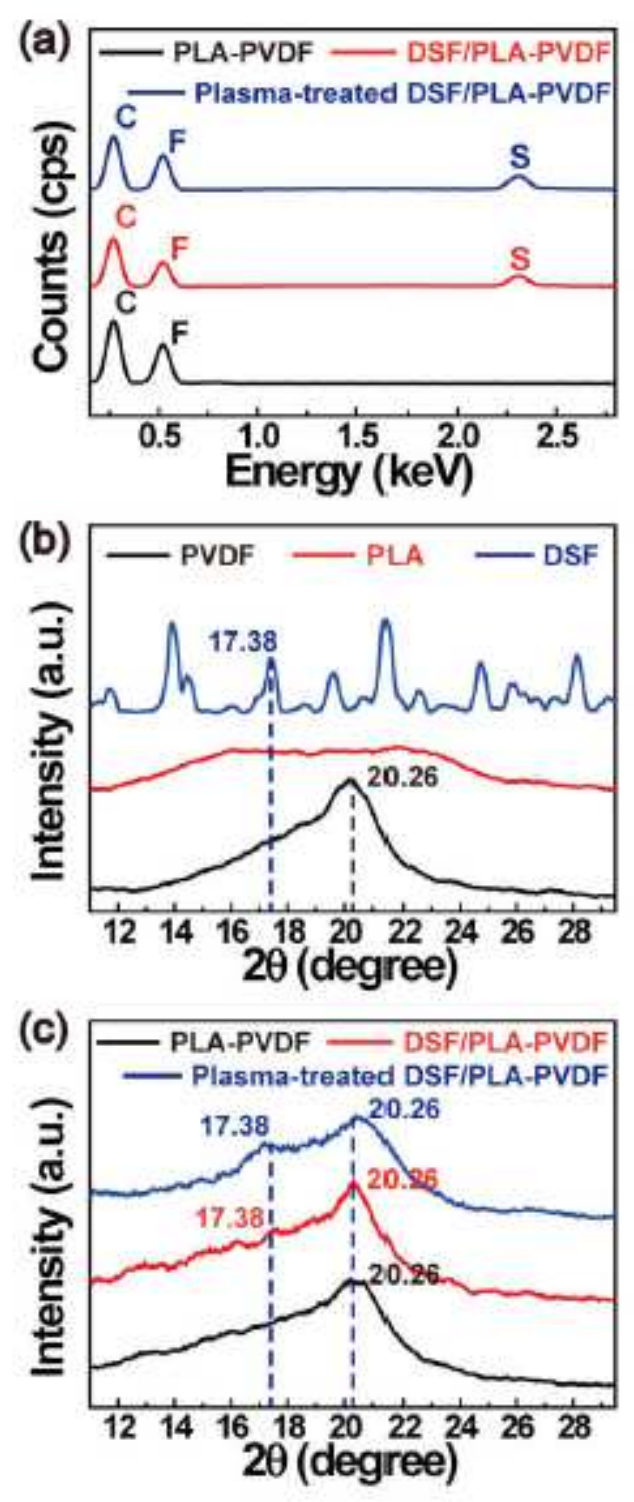

Figure 3

(a) EDS spectrum of the bi-layered fiber membranes. (b) XRD patterns of DSF powders, PLA fibers and PVDF fibers. (c) XRD patterns of PLA-PVDF, DSF/PLAPVDF and plasma-treated DSF/PLA-PVDF. 

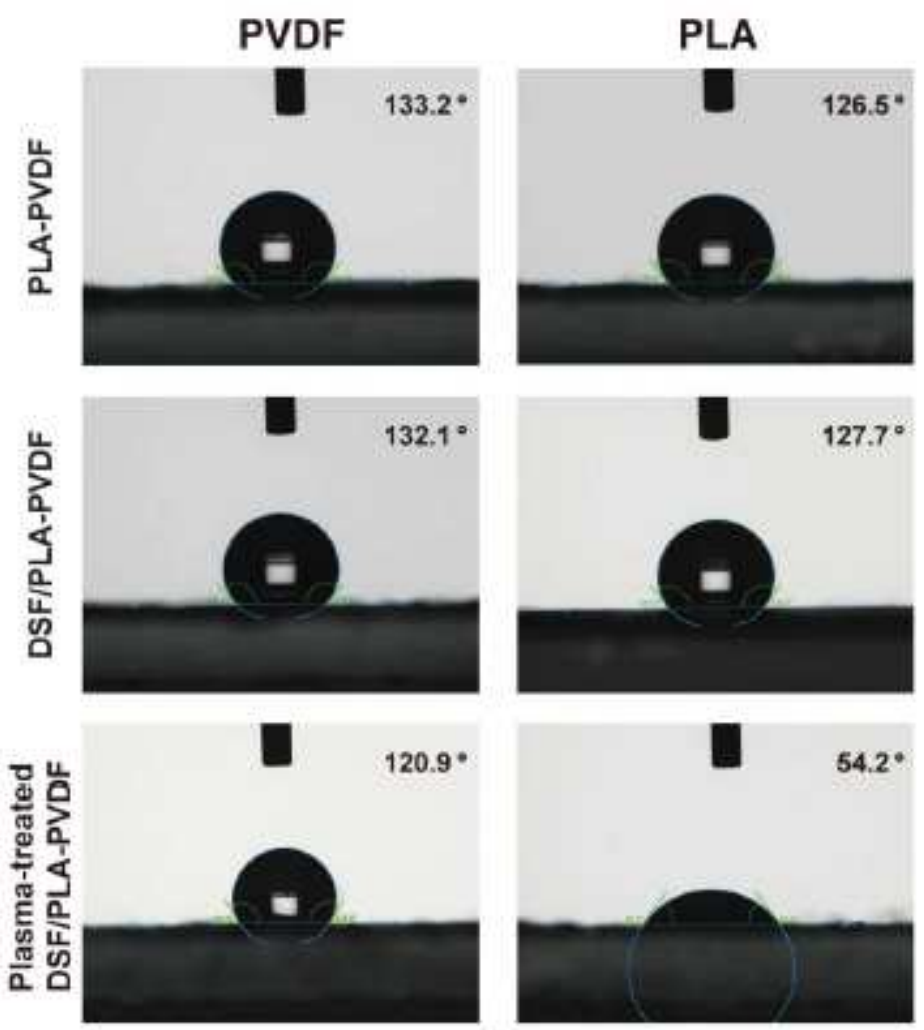

Figure 4

The top (PLA) and bottom (PVDF) surface water contact angles of PLA-PVDF, DSF/PLA-PVDF and plasma-treated DSF/PLA-PVDF.
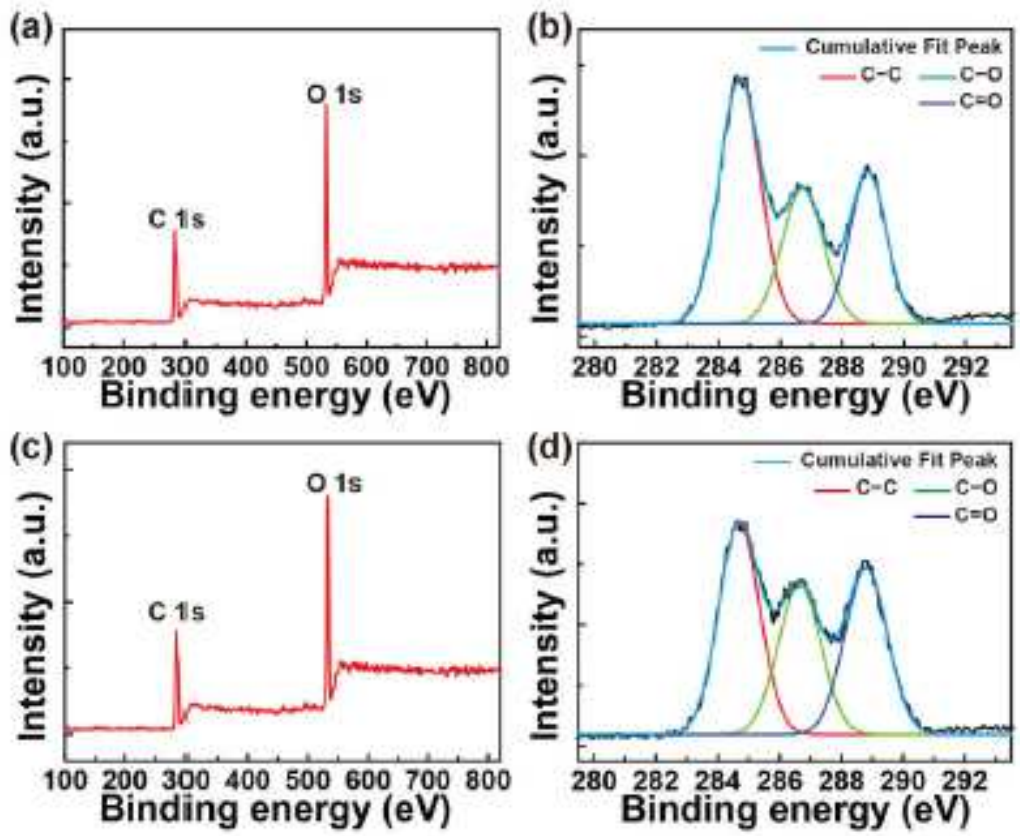

Figure 5 
(a), (b) XPS spectra survey scan and C1s XPS spectra of DSF/PLA-PVDF. (c), (d) The corresponding XPS measurement results of plasma-treated DSF/PLA-PVDF.
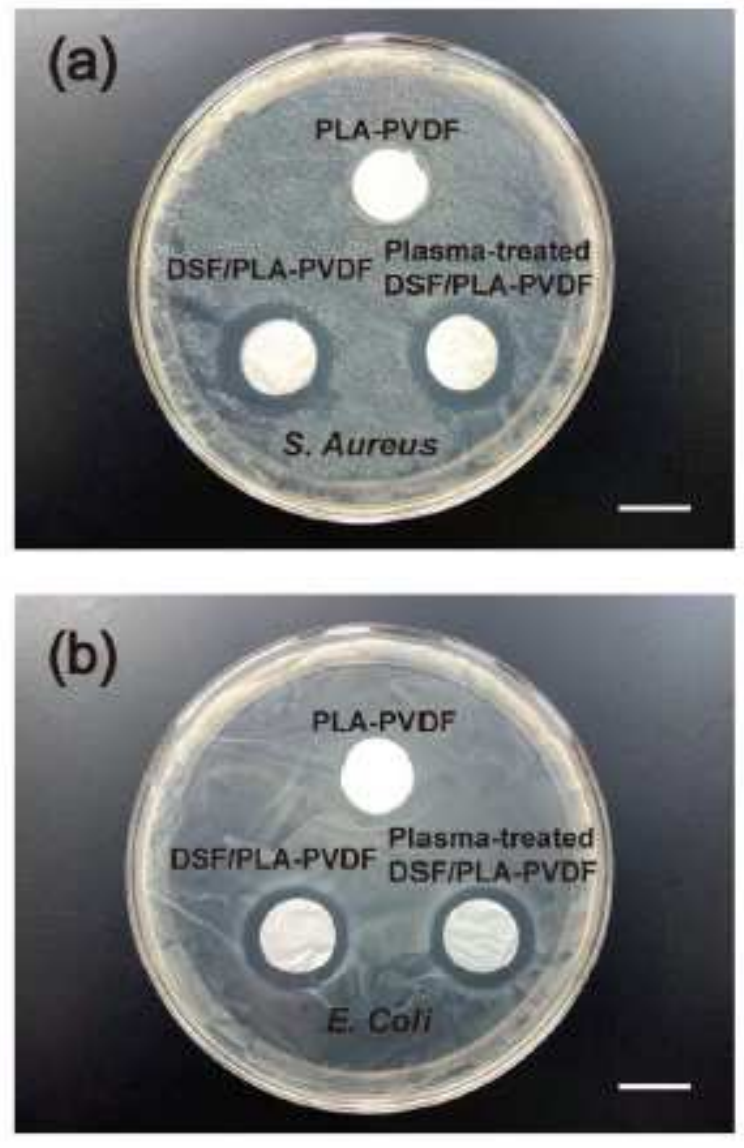

\section{Figure 6}

Inhibition zone of bi-layered fiber membranes against (a) S. Aureus and (b) E. Coli. (scale bar, $15 \mathrm{~mm}$ ) 